Sharif University of Technology
Scientia Iranica

\title{
Optimal winding selection for wound-rotor resolvers
}

\author{
M. Mohammad-Yari ${ }^{\mathrm{a}}$, M.R. Safari ${ }^{\mathrm{a}}$, R. Alipour-Sarabi ${ }^{\mathrm{a}}$, Z. Nasiri-Gheidari ${ }^{\mathrm{a}, *}$, \\ and F. Tootoonchian ${ }^{\mathrm{b}}$
}

a. Department of Electrical Engineering, Sharif University of Technology, Tehran, Iran.

b. Department of Electrical Engineering, Iran University of Science and Technology, Tehran, Iran.

Received 24 December 2018; received in revised form 20 May 2019; accepted 21 July 2019

\section{KEYWORDS}

Electromagnetic

sensors;

Winding arrangement;

Resolver;

Time-stepping finite

element analysis.

\begin{abstract}
Wound-Rotor (WR) resolvers are the most commercially used resolvers in industrial applications. In this regard, the present study evaluates the effect of different winding arrangements on the accuracy of WR resolvers. To this end, three windings were proposed for the stator of the resolver containing on-tooth overlapping winding, distributed lap winding, and distributed concentric winding. Those windings were also applied to the rotor. All the rotor windings were assumed to be single- and two-phased. In addition, the effect of damper winding was also evaluated in this study. The analysis was done using time-stepping finite element method, and the most accurate resolver was built and tested. Close agreement between the results from experimental measurements and finite element confirms the obtained results.
\end{abstract}

(C) 2021 Sharif University of Technology. All rights reserved.

\section{Introduction}

Resolvers are one of the most common position sensors in industrial applications. In industries with special conditions such as wide temperature variation, high vibration level, and polluted environments, resolvers are the most promising choice [1-3]. In such applications, the optical encoders, the only competitors of the resolvers in terms of accuracy, lose proper operation. However, compared to encoders, resolvers are costly. In this regard, Variable Reluctance (VR) resolvers have recently caught researchers' attention to be applied in some industries [4,5]. The rotor of VR resolvers is characterized by a solid structure without any winding. Therefore, there is no need for rotary transformer in VR resolvers. In other words, VR resolvers are more efficient than the conventional WR resolvers in terms of cost. However, the application

*. Corresponding author. Tel.: +982166164389 E-mail address: znasiri@sharif.edu (Z. Nasiri-Gheidari)

doi: $10.24200 /$ sci. 2019.52439 .2764 of VR resolvers is subject to several challenges. To discuss such challenges, the VR resolvers should be categorized first. These resolvers operate based on the sinusoidal variation of air-gap reluctance. In this regard, two types of VR resolvers emerge: Sinusoidal AirGap Length (SAGL) ([3-9]) and Sinusoidal Area (SA) resolvers [10-14]. The commercial VR resolvers are SAGL types. They suffer from inappropriate performance in two-pole applications and high accuracy deterioration under eccentricity faults. To overcome the first challenge in [8], a new structure is proposed for SAGL resolvers. Despite the acceptable accuracy of that resolver, it has an excessive axial length in comparison with that of conventional SAGL resolvers. To improve the resiliency of the SAGL resolver under eccentricities, disk type resolvers were proposed [3,9]. Furthermore, SA resolvers were also proposed to overcome the disadvantages of the SAGL resolvers [10]. However, they have their own drawbacks, i.e., huge dimensions and inappropriate performance under run-out fault. In [11,12], a new optimized structure was proposed to solve the problems. However, their proposed structure needs more improvements to be 
comparable with conventional WR resolvers from the accuracy point of view. Some other affordable two-pole resolvers were proposed in [15] that were not applicable in high-accuracy applications.

Finally, according to the authors' best knowledge, the most accurate commercial two-pole resolvers are still WR resolvers. Accordingly, this paper sets forth the accuracy improvement of two-pole WR resolvers. Numerous studies have been conducted on the performance improvement of WR resolvers [16-23]. For instance, in [17], the effect of physical parameters of a disk-type WR resolver on its accuracy was discussed. As shown, for a given number of slots, the estimated position error affects the pole numbers of resolvers. Increasing the number of poles ensures the higher accuracy of the resolver. However, mechanical difficulties hinder the manufactures' ability to increase the pole numbers by increasing the slot numbers. To overcome this constraint, in $[18,19]$, new winding methods including fractional slot concentrated winding were proposed for large-pole-number WR resolvers with a small number of slots. However, it seems that the accuracy of the estimated position can increase further. In this regard, in [2], the genetic algorithm was used to calculate the optimal winding of a high pole number disk type resolver. Moreover, in [20], it was suggested that damper winding be used to improve the performance of a disk-type resolver under eccentricities. The frequency response of a cylindrical resolver was presented in [21]. In [22], the performance of a cylindrical WR resolver under static, dynamic, and mixed eccentricities was evaluated. An analytical model based on the d-q axis theory was proposed in [23]. However, most of the mentioned studies have employed the distributed winding or fractional slot concentrated winding for their studied resolvers. The winding arrangement had significant influence on the performance of WR resolvers. In this respect, different winding methods were used for the stator and rotor of WR resolvers in this study, and the best one was selected for experimental evaluation based on the minimization of position error.

\section{The studied resolver}

The studied resolver is a two-pole WR cylindrical resolver containing 24 slots in the stator and 20 in the rotor. A Rotary Transformer (RT) is used to feed the excitation winding of the rotor. The primary winding of the RT is fed by a high-frequency sinusoidal voltage, and the induced voltage in the secondary coil of the RT located on the rotor is used to supply the excitation voltage of the rotor. There is single- or twophase winding on the rotor of the resolver. The first mandatory phase is called excitation winding which is connected to the secondary coil of RT. The next optional winding with a 90 -degree phase difference with respect to the excitation winding is a short-circuited winding, also called damper winding. The effect of the existence of the damper winding on the accuracy of the resolver was taken into account in this paper. The stator of the resolver is equipped with two-phase windings that have 90-degree phase difference with each other, called signal windings (sine and cosine windings). The signal windings are connected to a Resolver-to-Digital Converter (RDC) that has high input impedance [24]. In this respect, the signal windings can electrically be assumed as an open circuit. Figure 1 shows the stator and rotor of the studied resolver, and Table 1 presents its geometrical dimensions. Both of the stator and rotor cores are made of identical laminated ferromagnetic materials.

The present study mainly focuses on the winding arrangement of the stator and rotor. Different arrangements are suggested for the studied resolver in the next section.

To better clarify the position detection strategy, it is assumed that the primary coil of the RT is fed by an input voltage as shown in the following:

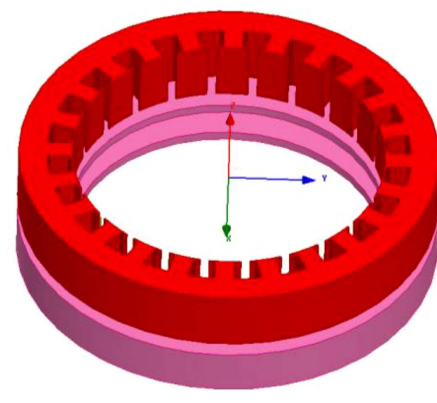

(a)

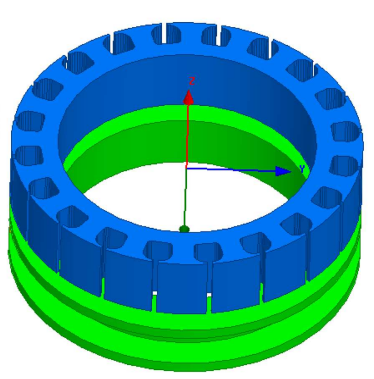

(b)
Figure 1. The studied resolver: (a) The stator core and the primary core of RT and (b) the secondary core of RT and the core of rotor.

Table 1. The geometrical dimensions and the excitation information of the studied resolver.

\begin{tabular}{lcc}
\hline Parameter & Unit & Value \\
\hline Slot numbers: stator/rotor & - & $24 / 20$ \\
Pole pairs & - & 1 \\
Air-gap length & $\mathrm{mm}$ & 1 \\
Stator/ rotor outer diameter & $\mathrm{mm}$ & $46 / 32$ \\
Stator slot opening width/height & $\mathrm{mm}$ & $1 / 0.2$ \\
Rotor slot opening width/height & $\mathrm{mm}$ & $0.5 / 0.3$ \\
Stator/rotor slot height & $\mathrm{mm}$ & $2 / 2.4$ \\
Stator/rotor slot width & $\mathrm{mm}$ & $3 / 2.4$ \\
core length: stator/rotor & $\mathrm{mm}$ & 6.7 \\
Rotational speed & $\mathrm{rpm}$ & 300 \\
Excitation voltage amplitude & $\mathrm{V}$ & 5 \\
Excitation frequency & $\mathrm{kHz}$ & 4 \\
\hline
\end{tabular}




$$
V_{R T 1}=V_{m} \cos (\omega t)
$$

where $V_{m}$ is the amplitude of the voltage fed to the primary coil of RT and $\omega$ is the angular frequency. The induced voltage in the secondary coil of RT $\left(V_{R T 2}\right)$ can be written as:

$$
V_{R T 2}=\frac{N_{2}}{N_{1}} V_{m} \cos (\omega t),
$$

where $N_{1}$ and $N_{2}$ are the numbers of primary and secondary coils turn, respectively. The excitation current $\left(I_{e x}\right)$ can be calculated as follows:

$$
\begin{aligned}
& I_{e x}=I_{\max } \cos (\omega t-\varphi), \\
& I_{\max }=\frac{N_{2} V_{m}}{N_{1} \sqrt{R_{e x}^{2}+L_{e x}^{2} \omega^{2}}}, \\
& \varphi=\operatorname{Arctan} \frac{L_{e x} \omega}{R_{e x}}
\end{aligned}
$$

where $R_{e x}$ and $L_{e x}$ are the resistance and inductance of the excitation winding, respectively. The induced voltage in the signal windings can be written as follows $[25]$ :

$$
\begin{aligned}
V_{a}= & -r_{s} i_{a}+M I_{e x} \frac{d \vartheta}{d t} \cos (\vartheta) \\
& -M I_{\max } \omega \sin (\vartheta) \sin (\omega t-\varphi), \\
V_{b}= & -r_{s} i_{b}-M I_{e x} \frac{d \vartheta}{d t} \sin (\vartheta) \\
& -M I_{\max } \omega \cos (\vartheta) \sin (\omega t-\varphi),
\end{aligned}
$$

where $V_{a}$ and $V_{b}$ are the phase voltages, $r_{s}$ the resistance of the signal winding, $M$ the mutual inductance between stator circuit and excitation winding, $\vartheta$ the rotor position; and $i_{a}$ and $i_{b}$ are the phase currents. The two first phrases in Eqs. (6) and (7) are negligible. Since the stator currents are almost zero and the excitation frequency $(\omega)$ is much higher than the rotor speed $(d \vartheta / d t)$, the rotor position $(\vartheta)$ can be calculated as follows:

$$
\vartheta=\tan ^{-1}\left(\frac{V_{a}}{V_{b}}\right) .
$$

A comparison of this position with the reference position yields the position error.

\section{Proposed winding arrangements}

Three different arrangements were examined for the stator winding namely Swdg1, Swdg2, and Swdg3. Those arrangements are shown in Figure 2. In this figure, Swdg1 is an overlapping on-tooth variable turn

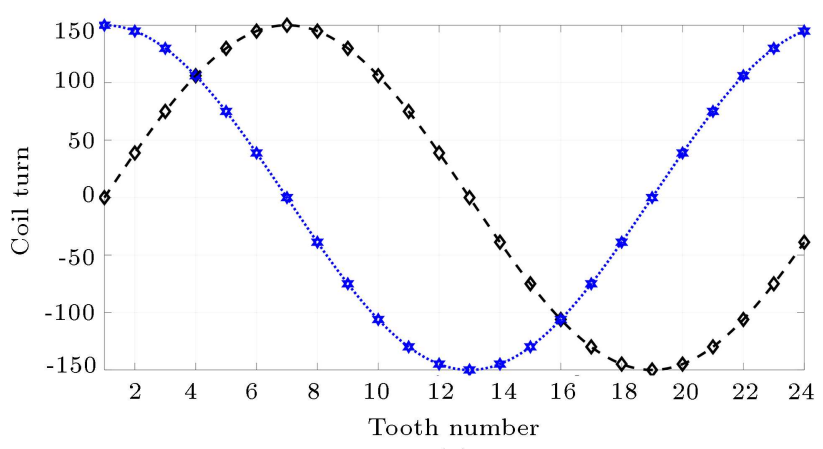

(a)

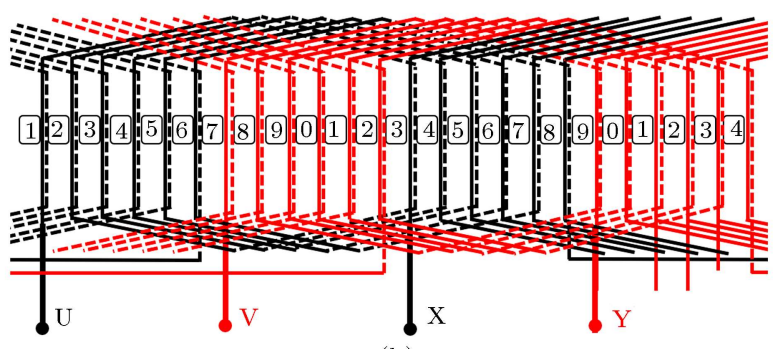

(b)

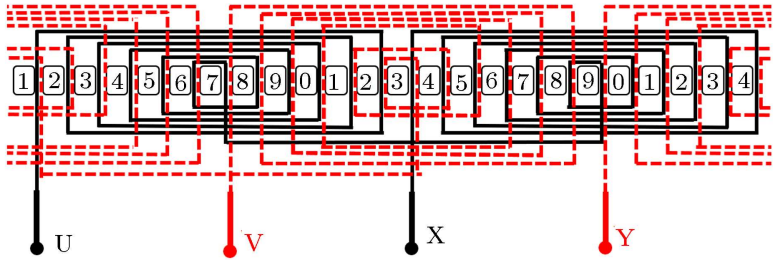

(c)

Figure 2. The proposed stator windings: (a) Swdg1, (b) Swdg2, and (c) Swdg3.

winding. The turns of sine and cosine coils on the $i$ th stator tooth are determined as follows [1]:

$$
\begin{aligned}
& N_{s . i}=N_{\max } \times \sin P_{w}\left[\frac{2 \pi}{Z}(i-1)\right], \\
& N_{c . i}=N_{\max } \times \cos P_{w}\left[\frac{2 \pi}{Z}(i-1)\right] .
\end{aligned}
$$

Here, $N_{\max }=150$ represents the maximum number of coil turns, $P_{w}=1$ the winding pole pair, and $Z=24$ the number of stator teeth. Although using different turns in each coil and overlapping configuration make Swdg1 practically too hard to be implemented, short overhang length and significant slot space usage are deemed as the merits of this configuration.

Swdg2 is a lap-distributed winding. Usually, in such a winding type, all the coils have identical coil pitch and turn numbers. Therefore, preparation of the coils for winding is easy. However, to improve the performance of the resolver in this paper, the sinusoidally distributed turn numbers are used for winding. Generally, it is believed that using distributed winding 
in electrical machines results in higher efficiency, higher amplitude of fundamental harmonic, and more desirable performance. These advantages should be evaluated for resolvers. However, Swdg2 has one drawback, i.e., its overhang length is longer than that of Swdg1.

Swdg3 is a concentric winding. The coil pitch and the turn number of coils are subject to variation in this configuration. It is also a common winding type in the commercial WR resolvers.

The above-mentioned three arrangements are considered for rotor as well. The proposed rotor windings, Rwdg1, Rwdg2, and Rwdg3, are listed in Figure 3. As mentioned in [20], using damper winding had no significant effect on the accuracy of the aligned resolver; however, under eccentricities, it could help improve the accuracy of faulty resolver. Consequently, despite the increasing copper usage, it is preferred to add the damper winding on the rotor of WR resolvers.

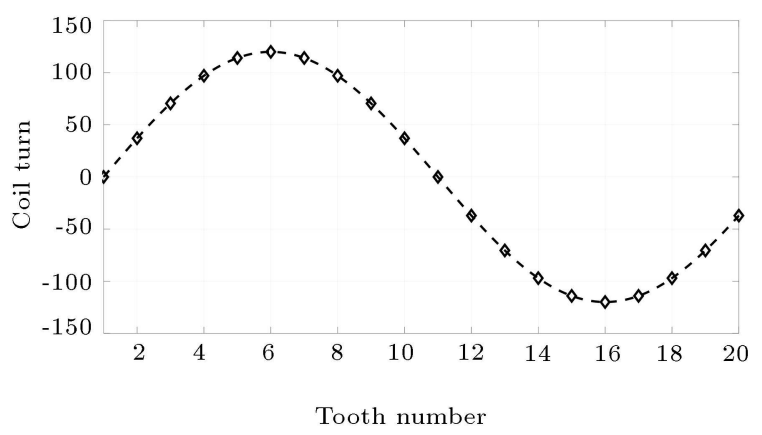

(a)

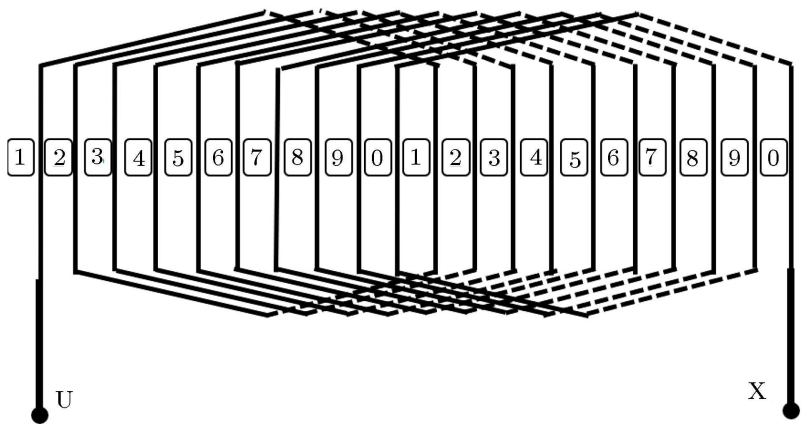

(b)

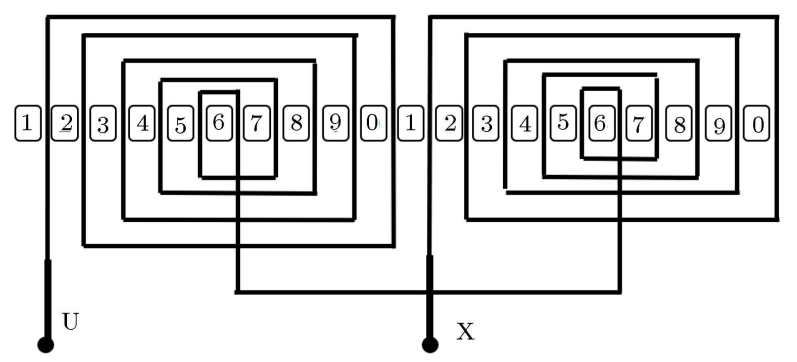

(c)

Figure 3. The proposed rotor windings: (a) Rwdg1, (b) Rwdg2, and (c) Rwdg3.

\section{Finite element analysis}

Time-stepping finite element analysis was employed to evaluate the proposed windings. To ensure that the employed mesh quality was good enough to obtain accurate results within a well-advised computation time duration, the optimal mesh was imported from magnetostatic solution to transient solution environment [26]. Furthermore, the distribution of magnetic flux density on the studied sensors was checked to ensure that the sensor was working in the linear part of the magnetization cure of the ferromagnetic material.

Another important point in resolver simulation is the correct selection of the time step and excitation frequency. The time step must be chosen such that at least 16 points would exist in one cycle of excitation frequency. Although both current source and voltage source are commonly used in the finite element simulation of resolvers, the last one is selected in this paper to take both space and time harmonics into account.

The simulations are categorized into two parts: without damper winding and with damper winding.

\subsection{Without using damper winding}

In this section, the simulation results for nine resolvers with the described windings listed in Table 2 are presented. Figure 4(a) shows the analogue voltages of Res1. As observed, the envelopes of the voltages are sine and cosine functions which are used for calculating the position. To calculate the envelope of the voltages, Hilbert transform was used in MATLAB software. Frequency analysis of the envelope presented in Figure 4(b) denotes the dominant harmonic in output voltage as the third one. Total Harmonic Distortion (THD) of the envelope is about $0.11 \%$. It should be mentioned that although low THD is a required term for the acceptable performance of a resolver, it is not an adequate condition, because it is devoid of sensitivity to harmonic order and the harmonic phase. So, it is required to calculate the

Table 2. Different combinations of stator and rotor windings.

\begin{tabular}{ccc}
\hline Resolver & Stator winding & Rotor winding \\
\hline Res1 & Swdg1 & Rwdg1 \\
Res2 & Swdg1 & Rwdg2 \\
Res3 & Swdg1 & Rwdg3 \\
Res4 & Swdg2 & Rwdg1 \\
Res5 & Swdg2 & Rwdg2 \\
Res6 & Swdg2 & Rwdg3 \\
Res7 & Swdg3 & Rwdg1 \\
Res8 & Swdg3 & Rwdg2 \\
Res9 & Swdg3 & Rwdg3 \\
\hline
\end{tabular}




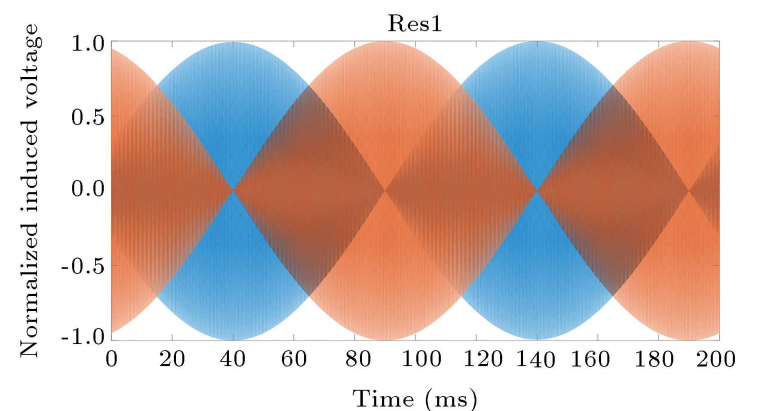

(a)

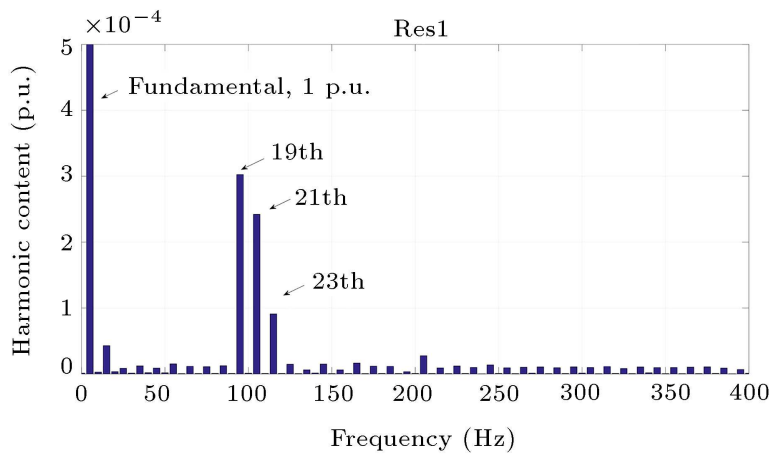

(b)

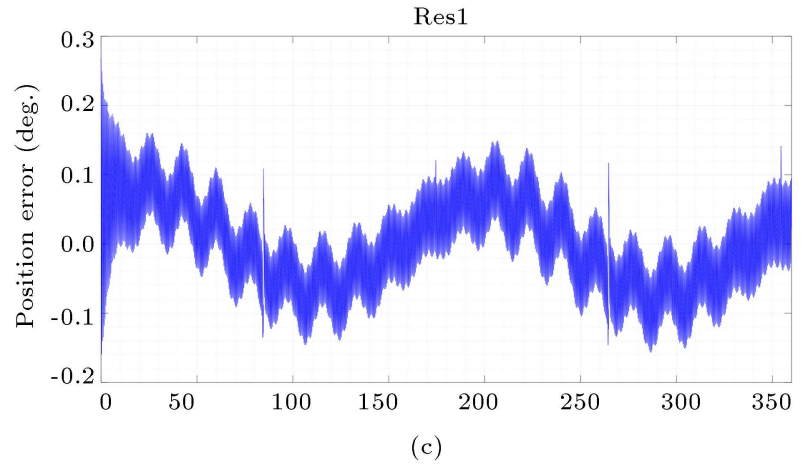

Figure 4. The results of finite element simulation of Res1: (a) The analogue voltages, (b) frequency spectrum of voltages' envelope, and (c) position error.

position error to ensure fair and accurate judgment about resolver's performance.

Figure 4(c) shows the position error of Res 1 . It can be seen that the Maximum Position Error (MPE) and the Average of Absolute Position Error (AAPE) are $0.2684^{\circ}$ and $0.0545^{\circ}$, respectively.

For the sake of brevity, the results of performance analysis of the other resolvers are reported in the next section along with those of resolvers with damper.

\subsection{With damper winding}

As mentioned earlier, damper winding is a short-circuit winding that has a 90-degree phase difference with respect to the excitation winding on the rotor. The rotor windings considering the damper winding are shown in Figure 5(a) to (c), called Rwdg4, Rwdg5, and Rwdg6, respectively. So, all the simulations are

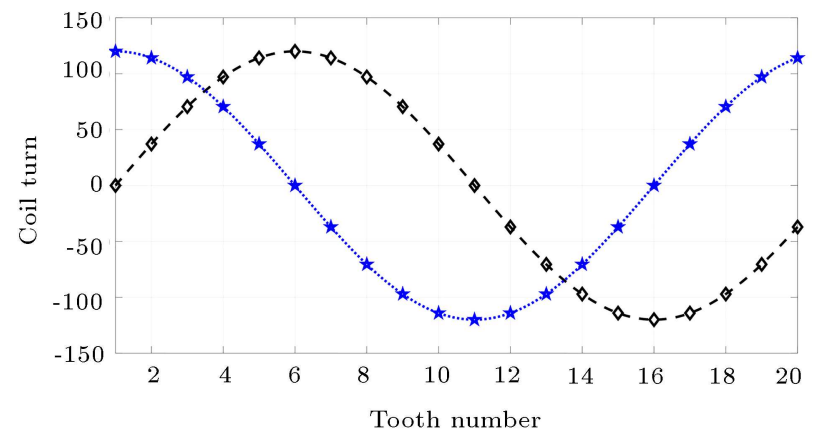

(a)

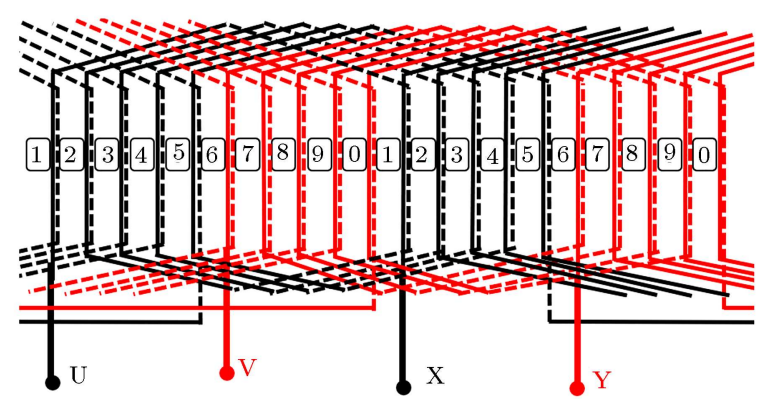

(b)

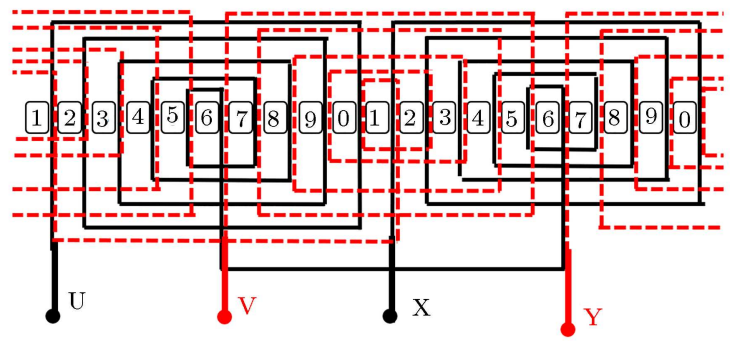

(c)

Figure 5. The proposed rotor windings equipped with damper winding: (a) Rwdg4, (b) Rwdg5, and (c) Rwdg6.

repeated with new rotor windings. Figure $6(\mathrm{a})$ to (c) compare the THD of voltages' envelopes, MPE, and AAPE of the Res1 through Res9 with and without damper winding. It can be seen that from THD point of view, the lowest THDs without damper winding belong to Res $9(0.076 \%)$ and Res $3(0.082 \%)$, respectively. Using damper winding improves the THD in all cases. However, when the stator has distributed lap winding and the rotor has distributed concentrated winding (Res6), the improvement is insignificant. On the other hand, Res9 with concentrated winding on both the stator and the rotor shows the highest improvement on the value of THD. The THD of Res 9 decreases from $0.076 \%$ to $0.035 \%$ after using damper winding. Comparison of the MPE of the resolvers before and after using damper winding in Figure 6(b) shows that the worst case is related to Res6 that is equipped with distributed lap winding on the stator and concentrated winding on the rotor. Furthermore, using concentrated winding on the stator with on-tooth or concentrated 


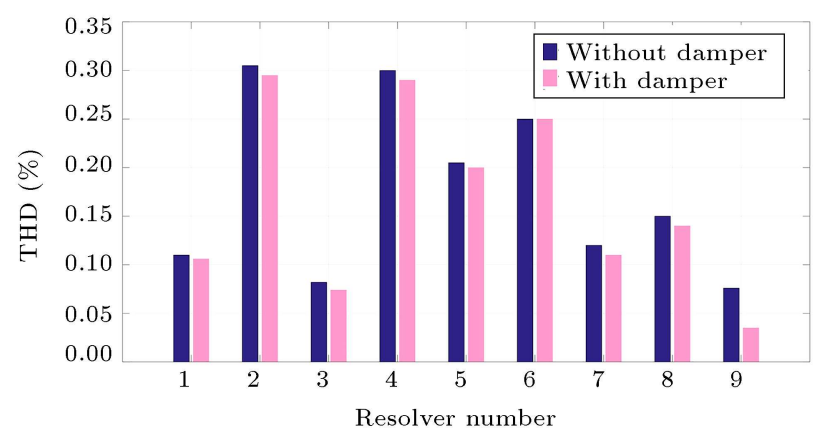

(a)

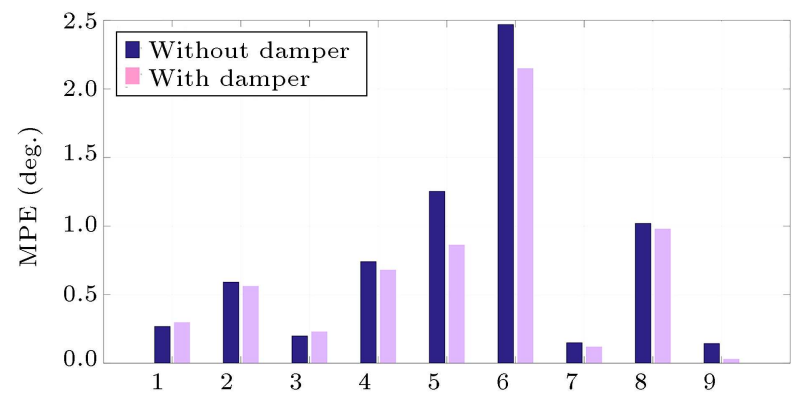

(b)

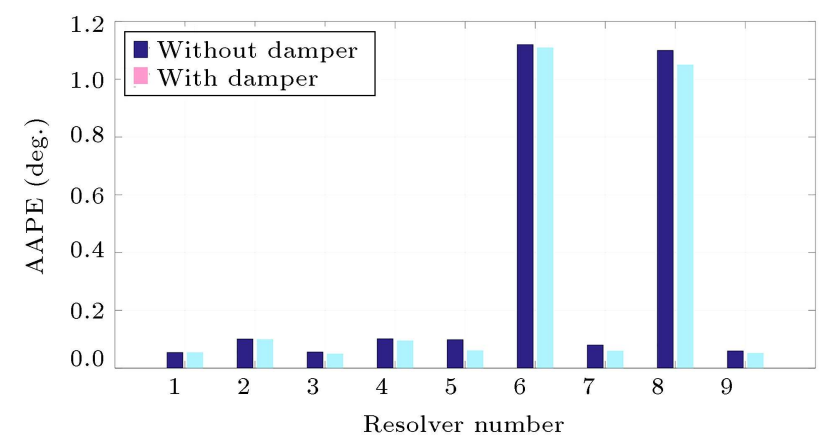

(c)

Figure 6. Comparing the performance of different resolvers with and without damper winding: (a) THD of voltages' envelope, (b) MPE, and (c) AAPE.

winding on rotor generates lower MPE than other winding combinations. However, it is worth mentioning that the best indicator for resolver accuracy is AAPE. Therefore, AAPEs of different resolvers are compared in Figure 6(c). It can be seen that the combination of lap and concentrated windings does not yield a promising result. The two most accurate resolvers without using damper winding are Res1 and Res3 with the AAPEs of $0.0545^{\circ}$ and $0.0556^{\circ}$, while the most accurate resolver after adding damper winding is Res3 with the AAPE of $0.0497^{\circ}$.

\section{Experimental evaluation}

The most accurate resolver (Res3, with damper winding) is chosen for experimental evaluation. The prototype of the sensor is built and the optimal winding

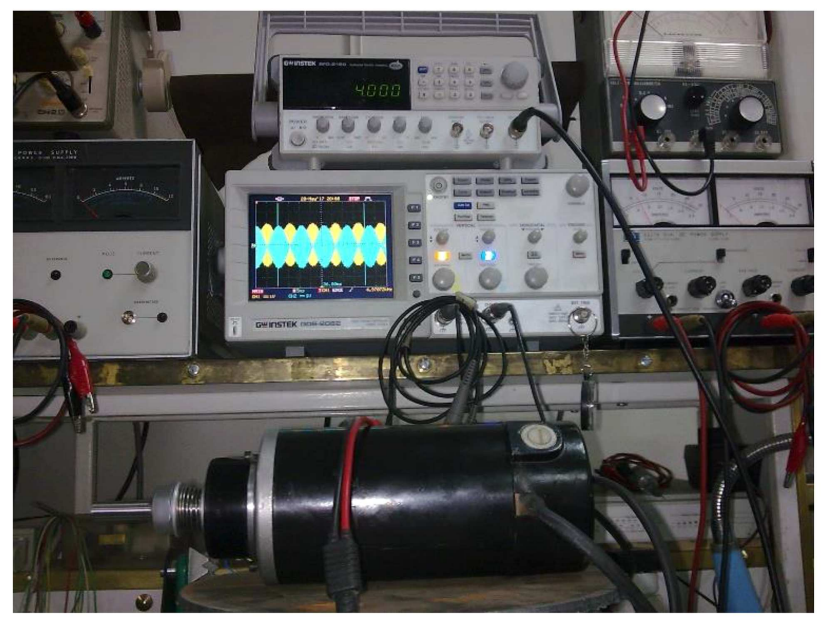

Figure 7. The test circuit of the studied resolver.

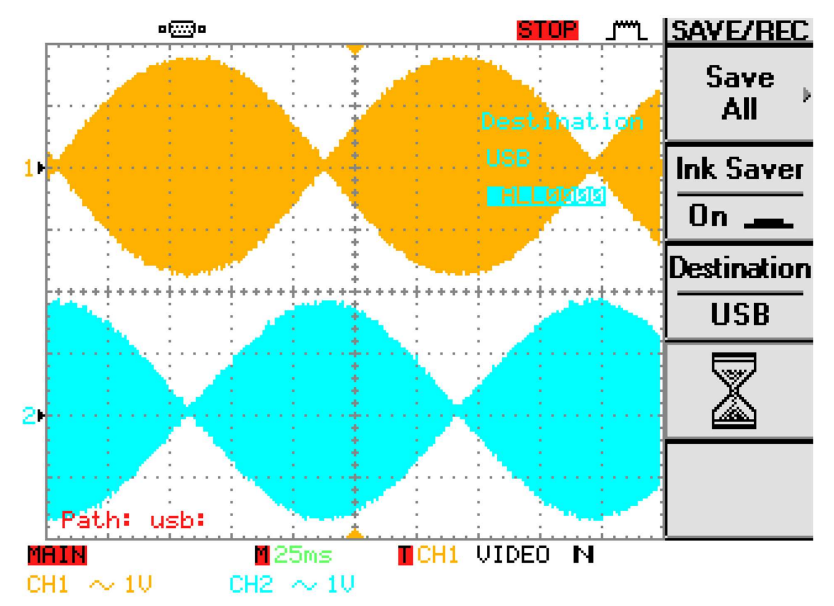

Figure 8. The analogue voltages of the prototype resolver.

is applied for measurements. Figure 7 shows the test circuit of the resolver where a DC motor is employed as a prime mover and a programmable optical encoder as a reference sensor. The output voltages of the resolver are saved and captured using a digital oscilloscope. The excitation voltage of the RT's primary coil is obtained from a digitally synthesized function generator.

The measured analogue voltages are shown in Figure 8. Similar to simulation results, the Hilbert transform is used to calculate the envelopes and the position error in off-line method. Table 3 shows THD of envelopes, MPE, and AAPE in comparison with those of simulation results. It can be seen that the error between the simulation and experimental results is less than 10\%, which confirms the accuracy of the simulations.

\section{Conclusion}

In this study, the effect of stator and rotor windings' arrangement on the accuracy of wound rotor cylindrical 
Table 3. Comparison between the simulation and measured results of Res3 with damper winding.

\begin{tabular}{ccc}
\hline & Measured & $\begin{array}{c}\text { Finite element } \\
\text { simulation }\end{array}$ \\
\hline THD (\%) & 0.082 & 0.0740 \\
MPE (deg.) & 0.252 & 0.2302 \\
AAPE (deg.) & 0.055 & 0.0497 \\
\hline
\end{tabular}

resolver was discussed. Three different windings were applied to the stator including on-tooth winding, lap winding, and concentrated winding. For rotor, the mentioned windings were applied with and without damper winding. Therefore, the effect of damper winding on the performance of the resolver was also clarified. Finally, it was shown that the highest improvement for the average of absolute position errors was achieved in the application of damper winding in Res3, which was equipped by both on-tooth winding on the stator and concentrated winding on the rotor.

Simulations were done using time stepping finite element analysis and verified by experimental measurement on the prototype of the most accurate resolver.

\section{References}

1. Abolqasemi-Kharanaq, F., Alipour-Sarabi, R., NasiriGheidari, Z., et al. "Magnetic equivalent circuit model for wound rotor resolver without rotary transformer's core", IEEE Sensors J., 18(21), pp. 8693-8700 (2018).

2. Alipour-Sarabi, R., Nasiri-Gheidari, Z., Tootoonchian, F., et al. "Improved winding proposal for wound rotor resolver using genetic algorithm and winding function approach", IEEE Trans. Industrial Electronics, 66(2), pp. 1325-1334 (2019).

3. Saneie, H., Nasiri-Gheidari, Z., and Tootoonchian, F. "Design-oriented modelling of axial-flux variablereluctance resolver based on magnetic equivalent circuits and Schwarz-Christoffel mapping", IEEE Trans. Industrial Electronics, 65(5), pp. 422-4330 (2018).

4. Ge, X., Zhu, Z.Q., Ren, R., et al. "A novel variable reluctance resolver for $\mathrm{HEV} / \mathrm{EV}$ applications", IEEE Trans. Ind. Appl., 52(4), pp. 2872-2880 (2016).

5. Ge, X., Zhu, Z.Q., Ren, R., et al. "A novel variable reluctance resolver with nonoverlapping tooth-coil windings", IEEE Trans. Energy Conversion, 30(2), pp. 784-794 (2015).

6. Daniar, A., Nasiri-Gheidari, Z., and Tootoonchian, F. "Performance analysis of linear variable reluctance resolvers based on improved winding function approach", IEEE Trans. Energy Convers., 33(3), pp. 1422-1430 (2018).

7. Daniar, A. and Nasiri-Gheidari, Z. "The influence of different configurations on position error of linear variable reluctance resolvers", 25th Iranian Conference on Electrical Engineering (ICEE), Tehran, Iran, pp. 955-960 (2017).

8. Tootoonchian, F. and Nasiri-Gheidari, Z. "Twelveslot two-saliency variable reluctance resolver with nonoverlapping signal windings and axial flux excitation", IET Electric Power Applications, 11(1), pp. 49-62 (2017).

9. Nasiri-Gheidari, Z. and Tootoonchian, F. "An optimized axial flux variable reluctance resolver with concentric windings", 24th Iranian Conference on Electrical Engineering (ICEE), Shiraz, Iran, pp. 12841290 (2016).

10. Shang, H., Wang, M., Chen, N., et al. "The effects of stator and rotor eccentricities on measurement accuracy of axial flux variable-reluctance resolver with sinusoidal rotor", 17th International Conference on Electrical Machines and Systems (ICEMS), Hangzhou, China, pp. 1206-1209 (2014).

11. Nasiri-Gheidari, Z., Alipour-Sarabi, R., Tootoonchian, F., et al. "Performance evaluation of disk type variable reluctance resolvers", IEEE Sensors J., 17(13), pp. 4037-4045 (2017).

12. Nasiri-Gheidari, Z., Tootoonchian, F., and Zare, F. "Design oriented technique for mitigating position error due to shaft run-out in sinusoidal-rotor variable reluctance resolvers", IET Electric Power Applications, 11(1), pp. 132-141 (2017).

13. Bahari, M. and Nasiri-Gheidari, Z. "Longitudinal end effect in variable area linear resolver and its compensating methods", 26th Iranian Conference on Electrical Engineering (ICEE), Mashhad, Iran, pp. 1316-1321 (2018).

14. Zare, F., Nasiri-Gheidari, Z., and Tootoonchian, F. "The effect of winding arrangements on measurement accuracy of sinusoidal rotor resolver under fault condition", Measurement, 131, pp. 162-172 (2019).

15. Tootoonchian, F. "Proposal of a new affordable 2pole resolver and comparing its performance with conventional wound-rotor and VR resolvers", IEEE Sensors J., 18(13), pp. 5284-5290 (2018).

16. Daniar, A., Nasiri-Gheidari, Z., and Tootoonchian, F. "Position error calculation of linear resolver under mechanical fault conditions", in IET Science, Measurement \& Technology, 11(7), pp. 948-954 (2017).

17. Alipour-Sarabi, R., Nasiri-Gheidari, Z., Tootoonchian, F., et al. "Effects of physical parameters on the accuracy of axial flux resolvers", IEEE Trans. Magn., 53(4), pp. 1-11 (2017).

18. Alipour-Sarabi, R., Nasiri-Gheidari, Z., Tootoonchian, F., et al. "Performance analysis of concentrated wound-rotor resolver for its applications in high pole number permanent magnet motors", IEEE Sensors J., $\mathbf{1 7}(23)$, pp. $7877-7885$ (2017).

19. Alipour-Sarabi, R., Nasiri-Gheidari, Z., Tootoonchian, 
F., et al. "Analysis of winding configurations and slotpole combinations in fractional-slots resolvers", IEEE Sensors Journal, 17(14), pp. 4420-4428( 2017).

20. Nasiri-Geidari, Z. and Tootoonchian, F. "Axial flux resolver design techniques for minimizing position error due to static eccentricities", IEEE Sensors Journal, 15(7), pp. 4027-4034 (2015).

21. Figueiredo, J. "Resolver models for manufacturing", IEEE Trans. Industrial Electronics, 58(8), pp. 36933700 (2011).

22. Tootoonchian, F. "Effect of damper winding on accuracy of wound-rotor resolver under static-, dynamic and mixed-eccentricities", IET Electric Power Applications, 12(6), pp. 845-851 (2018).

23. Tootoonchian, F., Abbaszadeh, K., and Ardebili, M. "Novel axial flux brushless resolver analysis and optimization using 3D finite element and DQ model methods", Iranian Journal of Electrical and Electronic Engineering, 8(3), pp. 243-258 (2012).

24. Nasiri-Gheidari, Z. "Design, performance analysis, and prototyping of linear resolvers", IEEE Trans. Energy Convers., 32(4), pp. 1376-1385 (2017).

25. Saneie, H., Nasiri-Gheidari, Z., and Tootoonchian, F. "Analytical model for performance prediction of linear resolver", IET Electric Power Applications, 11(8), pp. 1457-1465 (2017).

26. Saneie, H., Alipour-Sarabi, R., Nasiri-Gheidari, Z., and Tootoonchian, F. "Challenges of finite element analysis of resolvers", In IEEE Transactions on Energy Conversion, 34(2), pp. 973-983 (2019). DOI: 10.1109/TEC.2018.2881465

\section{Biographies}

Mohammad Mohammad-Yari was born in Tehran, Iran in 1995. He received the BSc degree from Sharif University of Technology, Tehran, Iran in 2018. He is currently working toward the MSc degree in Electrical Engineering. His current research interests include design and optimization of electrical machines and electromagnetic sensors.
Mohammad Reza Safari was born in Tehran, Iran in 1996. He received the BSc degree in Electrical Engineering from Sharif University of Technology, Tehran, in 2019. He is currently working toward the MSc degree in Cognitive Science at Institute for Cognitive Science Study, Tehran, Iran. His current research interests include the assessment of cognitive capabilities from EEG data and BCI.

Ramin Alipour-Sarabi was born in Sarab, Iran in 1989. He received the $\mathrm{BSc}$ degree from Iran University of Science and Technology, Tehran, Iran in 2012 and MSc degree from the Sharif University of Technology, Tehran in 2014, where he is currently working toward the $\mathrm{PhD}$ degree, all in Electrical Engineering. His current research interests include power electronics and design, optimization, and performance analysis of electrical machines and electromagnetic sensors.

Zahra Nasiri-Gheidari received the BSc degree from the Iran University of Sciences and Technology, Tehran, Iran, in 2004 and the MS and PhD degrees from University of Tehran, Tehran in 2006 and 2012, respectively, all in Electrical Engineering. She is currently an Associate Professor at the Department of Electrical Engineering, Sharif University of Technology. Her research interests include design, optimization, and performance analysis of electrical machines and electromagnetic sensors.

Farid Tootoonchian received the BSc and MSc degrees in Electrical Engineering from the Iran University of Sciences and Technology, Tehran, Iran in 2000 and 2007 , respectively, and the $\mathrm{PhD}$ degree from the K. N. Toosi University of Technology, Tehran in 2012. He is currently an Associate Professor at the Department of Electrical Engineering, Iran University of Sciences and Technology. His research interests include design, optimization, finite-element analysis, and prototyping of ultrahigh-speed electrical machines and ultrahighprecision electromagnetic sensors. 